\section{Fact and language}

Linguistics and Injormation Science. By Karen Sparck Jones and Martin Kay. Pp. xii +244 . (Library and Information Science Series.) (Academic: New York and London, January 1974.) $\$ 14.50 ; £ 6.80$.

THis book is a state-of-the-art survey on the use of linguistic theories and techniques in information retrieval. The emphasis is on automated methods; that is, on liguistic theories that have been (or could be) used to develop computational methods of analysing language, and on their use in automated information retrieval systems. The survey concentrates on the Iiterature of 1965-1970.

The authors first take a bird's-eye view of activity in the separate fields of information retrieval and linguistics, and then consider the particular aspects of information retrieval which have an apparent linguistic content. The meat of the book comes in two chapters on syntax and semantics respectively, where a detailed analysis of the present state of linguistic theory is followed by a discussion of how this theory has been, or might be, applied to information retrieval. There is a further section on fact retrieval.

The authors lean fairly heavily on an analysis of the retrieval process which identifies three stages: informal interpretation of the document, formal representation of this interpretation, and manipulation of the representation in searching. One can imagine other views of the process (indeed, they mention another)-not necessarily better ones, but ones which emphasise other aspects. In some instances the authors are restricted by their particular approach. For example, they point out quite rightly that most comparative experiments that have been carried out with index descriptions using syntax seem to show that this syntax is not particularly useful in retrieval. But they do not consider one possibility: that the fact that the indexer (man or machine) has to formulate the description in a syntactically coherent form may influence his choice of terms, and hence may affect retrieval performance even if the terms are searched in a postcoordinate manner, ignoring syntax.

But this argument serves to reinforce one of the main conclusions of the book: that there is a lack of suitable theories of information retrieval which would indicate where linguistic theories might be of use. Overall, the conclusions are somewhat negative: both fields have to develop further before the one is likely to be a major contributor to the other. Indeed, there is some doubt that this will ever happen; but the authors argue persuasively that there are still plenty of possibilities.

The book performs its function as a survey very well, with a surprisingly good international coverage. It is coherent and well thought out, and also well written. The production is decidedly cheap-looking (yellow paper, a number of misprints, badly aligned type and uneven type density). But in content the book is a valuable contribution to the literature, which should help to bring order to a somewhat fragmented field. S. E. RoBERTSON

\section{Why plans go awry}

Planning and Budgeting in Poor Countries. By Naiomi Caiden and Aaron Wildavsky. Pp. xvii +371 . (Comparative Studies in Behavioural Science.) (Wiley: New York and London, March 1974.) £8.40.

A MAIN theme of this book had been anticipated by Bert Brecht in the Threepenny Opera. Freely translated, the song goes something like this:

Well, go and make a plan!

Seek where matrices lurk

Then go and make a second plan, Neither of them will work.

Or, in the words of the Nepalese planner, summarising the experience of his country: "Try one form of organization; no result. Try something else; no result. The result is always the same" (Page 216).

The two authors of this book are political scientists who have covered more than 80 poor countries with questionnaires and interviews in order to examine government planning and budgeting and to recommend reforms. Their work reflects the disenchantment with planning.

Some of the criticisms of plans as ambitious, abstract, irrelevant and unrealistic miss the point. The purpose of formal models is to trace the indirect effects of alternative policies. Things often turn out differently in the second and third round from the intentions of the first. First-round socialism may turn into third-round monopoly capitalism. Particularly but not only in poor countries good causes tend to be turned into rackets. The reason why planning has failed, where it has failed, is not that it uses abstract models and traces interdependence between variables, but that it has sometimes been pseudo-planning, confined to a ceremonial superstructure, without being geared to where the action is.

The authors argue for the central importance of the annual budget. Though it is obviously true that no plan can be implemented unless it is integrated into the annual budget, there is a danger of mistaking a nec- essary, though minor, condition for the strategic one. Budgets are, at best, annual public expenditure plans. A focus on fiscal magnitudes, though essential for proper public accounting, obscures and evades the real activities. Links between fiscal (or even financial) expenditures and results are tenuous, especially in underdeveloped countries. There are no fixed coefficients between money expenditure and land reform, population policy, incomes policy, education, public health, nutrition. Foreign exchange budgeting, manpower budgeting, raw material budgeting are just as important as fiscal budgeting and even they do not exhaust the range of necessary policies. Proper public accounting is necessary to ensure, negatively, that public money is not spent extravagantly or corruptly, but it cannot ensure, positively, that it is spent according to social priorities and that the necessary complementary actions are taken. Budgeting is to planning what bookkeeping is to business management: without it, management is impossible; but with the best book-keeper in the world, a firm can go bankrupt. These points are not enough stressed by the authors, who see the plan essentially as a many-year public capital budget.

Much is made of the need for redundancy. In a piece of exquisite jargon, the authors write: "Broadly speaking, we can regard social poverty as a lack of functional redundancy" (Page 49). But there is a vast difference between reserves (which serve a purpose) and redundancies. A more analytical and quantitative approach would have made the distinction clear. It is now well known that in poor societies, not only unskilled labour, but also capital and technically trained professional manpower are redundant: but, alas, they are not reserves.

The authors make a large number of entirely fair and commonsensical criticisms of planning. "If we were asked to design a mechanism for decisions to maximise every known disability and minimise any possible advantage of poor countries, we could hardly do better than comprehensive, multi-sectoral planning" (Page 293). The need for unavailable information, for political stability, for consistent aims are cited as unattainable conditions. But perhaps the most serious criticism of planning is omitted, viz. that its very success, measured by coherence and consistency, becomes an obstacle to adaptation and innovation. Plans introduce an additional rigidity into societies already inflexible. Plans, for this reason, in spite of their declared intentions, are elements strengthening conservatism.

The conclusion is not, however, reliance on laissez-faire and the free play 